NASA/TM-2003-212163

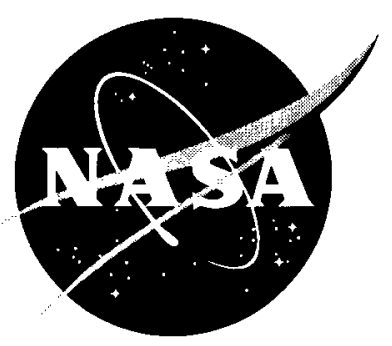

\title{
Computational Materials: \\ Modeling and Simulation of Nanostructured Materials and Systems
}

Thomas S. Gates and Jeffrey A. Hinkley

Langley Research Center, Hampton, Virginia

National Aeronautics and

Space Administration

Langley Research Center

Hampton, Virginia 23681-2199 
Available from:

NASA Center for AeroSpace Information (CASI)

7121 Standard Drive

Hanover, MD 21076-1320

(301) 621-0390
National Technical Information Service (NTIS) 5285 Port Royal Road

Springfield, VA 22161-2171

(703) 605-6000 


\begin{abstract}
The paper provides details on the structure and implementation of the Computational Materials Program at the NASA Langley Research Center. Examples are given that illustrate the suggested approaches to predicting the behavior and influencing the design of nanostructured materials such as high-performance polymers, composites, and nanotube-reinforced polymers. Primary simulation and measurement methods applicable to multi-scale modeling are outlined. Key challenges including verification and validation of models are highlighted and discussed within the context of NASA's broad mission objectives.
\end{abstract}

\title{
Introduction
}

"I have not failed. I've just found 10,000 ways that don't work." -Thomas Alva Edison (1847-1931)

Each distinct age in the development of humankind has been associated with advances in materials technology. Historians have linked key technological and societal events with the materials technology that was prevalent during the "stone age," "bronze age," and so forth. The description of our current age and culture will be up to future historians, but the last 350 years have seen many advances in materials technology that have helped shape our world today. Much of this groundbreaking work (Table 1) was because of perseverant research scientists and engineers finding solutions after long periods of experimentation and development.

Within the last 20 years, several research institutions have recognized the need for a more systematic approach to new materials development. This approach was one that would combine interdisciplinary research, new advances in computational modeling and simulation, and critical laboratory experiments to rapidly reduce the time from concept to end product. These institutions felt that this was the new paradigm by which all future materials research would be conducted and have embraced the concepts that have come to be known simply as "Computational Materials."

Traditionally, research institutions have relied on a discipline-oriented approach to material development and design with new materials. It is recognized, however, that within the scope of materials and structures research, the breadth of length and time scales may range more than 12 orders of magnitude, and different scientific and engineering disciplines are involved at each level. To help address this wide-ranging interdisciplinary research, Computational Materials at the NASA Langley Research center (LaRC) has been formulated with the specific goal of exploiting the tremendous physical and mechanical properties of new nano-materials by understanding materials at atomic, molecular, and supramolecular levels.

Computational Materials at LaRC draws from physics and chemistry, but focuses on constitutive descriptions of materials that are useful in formulating macroscopic models of material performance. The purpose of this paper is to describe in some detail how convergent technologies have facilitated the LaRC Computational Materials approach for aerospace materials and structures research. In particular, the paper discusses how the Computational Materials program at LaRC proposes to utilize multi-scale analysis methods and critical experiments to establish the technology for the scale-up of nanostructured materials into engineering level, multifunctional materials for advanced aircraft and spacecraft.

The benefits of the Computational Materials approach are three fold. First, it encourages a reduced reliance on costly trial and error, or serendipity, of the "Edisonian" approach to materials research. Second, it increases the confidence that new materials will possess the desired properties when scaled up from the laboratory level, so that lead-time for the introduction of new technologies is reduced. Third, the Computational Materials approach lowers the likelihood of conservative or compromised designs that might have resulted from reliance on less-than-perfect materials.

The paper is organized as follows. Key challenges are discussed and put into context of the broad challenges for NASA. Contributions from convergent technologies: biology, measurement science, and 
information technology, are presented. Details of the primary simulation methods are outlined and the issues of method verification and validation are explained.

\section{NASA Challenges}

NASA as an agency faces many technology challenges in the years ahead. Some of these broad challenges are directly related to the goals of the Computational Materials program. Most notable of these challenges are enhancing the performance of advanced aircraft and spacecraft by increasing: size per mass, strength per mass, function per mass and power, and intelligence per mass and power.

In terms of Computational Materials and the application of advanced nanostructured materials, these challenges translate into more specific requirements that include high-strength-per-mass smart materials for vehicles and large space structures, materials with designed-in mechanical/thermal/electrical properties, materials for high-efficiency energy conversion, and materials with embedded sensing/compensating systems for reliability and safety.

\section{Computational Materials}

In order to address these goals and challenges, the Computational Materials program has developed a scheme for spanning both the length and time scales associated with analyses that describe material behavior. Schematically, this approach is illustrated in Figure 1. The starting point is a quantum description of materials; this is carried forward to an atomistic scale for initial model development. Models at this scale are based on molecular mechanics or molecular dynamics. At the next scale, the models can incorporate micro-scale features and simplified constitutive relationships. Further progress up the scale leads to the meso or in-between levels that rely on combinations of micromechanics and wellestablished theories such as elasticity. The last step towards engineering-level performance is to move from mechanics of materials to structural mechanics by using methods that rely on empirical data, constitutive models, and fundamental mechanics. The central part of this hierarchical scheme, connections between the nano and micro scales, are examined in greater detail subsequently.

\section{Nanostructured Materials}

The origins of focused research into nanostructured materials can be traced back to a seminal lecture given by Richard Feynman in 1959[1]. In this lecture, he proposed an approach to "the problem of manipulating and controlling things on a small scale." The scale he referred to was not the microscopic scale that was familiar to scientists of the day but the unexplored atomistic scale. Over the subsequent years, this idea was refined and eventually resulted in the announcement of the National Nanotechnology Initiative in 2000[2]. It is ironic that in Feynman's lecture he conjectured that "in the year 2000, when they look back at this age, they will wonder why it was not until the year 1960 that anybody began seriously to move in this direction."

The recent history of "nano" science and engineering includes investigations into a variety of material systems and applications[3]. Table 1 highlights the discoveries of "buckyballs" (the $\mathrm{C}_{60}$ family) in 1985[4] and carbon nanotubes in 1991[5]. The nanostructured materials based on carbon nanotubes and related carbon structures are of current interest for NASA and much of the materials community. Although at the time of their discoveries, other materials with well-defined nanoscopic structure were known, investigators were intrigued to find that these new forms of carbon could be viewed as either individual molecules or as potential structural materials[6]. This realization in turn energized a whole new culture of nanotechnology research accompanied by worldwide efforts to synthesize nano-materials and to use them to create multifunctional composite materials. More broadly then, nanotechnology presents the vision of working at the molecular level, atom by atom, to create large structures with fundamentally new molecular organization. With regards to NASA's objectives within the National Nanotechnology Initiative, the goals include advances in ultralight, ultrastrong, space durable materials for very large space structures (telescopes, antennas, solar sails), spacecraft electronics for greater autonomy and on- 
board decision-making, micro systems based on biological principles, utilization of in-situ resources to create complex structures in space, and biologically-inspired architectures for long duration missions.

\section{Convergent Technologies}

The growth of Computational Materials research, with its emphasis on the concepts of nanotechnology and a hierarchical, multi-scale modeling approach, has relied to some extent on inspiration and advances in three key technology areas: biology or biomimetics, measurement science, and information technology. The convergence of these key technologies may provide the means for Computational Materials to eventually solve some of the most fundamental problems in materials science and engineering.

\section{Biological Inspiration}

To some extent, the emergence of Computational Materials as an approach to development and acceptance of new materials can find a parallel in the rapid growth of modeling and test in biotechnology over the last 50 years. A convenient marker to use as the start of this biotechnology revolution is the discovery of the structure and replication of DNA by Watson and Crick in 1953[7]. It can be argued that this research has been some of the most important biological work of the last 100 years, and the field it opened may be the scientific frontier for the next 100 years. Rapid advances in this field led to the official start of the Human Genome project in 1990[8]. Concurrently, advances in genetics and proteomics have provided revolutionary insights into the function and self-assembly of biological structures. Many of the modeling and characterization methods developed for biological materials have now found a prominent place within the broader materials community.

\section{Measurement Science}

Microscopy has consistently been a primary source of information on the fundamental structure of materials. Prior to the 1940's, microscopy was limited in resolution by the wavelength of visible light (approximately $10^{-6}$ to $10^{-7}$ meters) and the associated optics systems. The practical limitations of light microscopes are $500 \mathrm{x}$ to $1000 \mathrm{x}$ magnification and a resolution of 0.2 micrometers. Obviously, disceming the intrinsic structure of nano-scale materials is impossible at this resolution.

The discovery of the Transmission Electron Microscope (TEM) occurred in the early 1940's and the first commercial electron microscopes became available around 1965. The Scanning Electron Microscope (SEM) is a microscope that uses electrons rather than light to form an image by scanning the beam across the specimen. The typical SEM has a magnification range from $15 x$ to $200,000 x$ and a resolution of 5 nanometers.

Beginning with the scanning tunneling microscope (STM) in 1981, experimentalists developed new techniques and devices for discerning the most basic unit of materials, the atom. Instruments that use variations of the principles of the STM are often called scanning probe microscopes (SPM). All of these microscopes work by measuring a local property -such as height, optical absorption, or magnetism - with a probe or "tip" placed very close to the sample. The small probe-sample separation (on the order of the instrument's resolution) makes possible for the first time imaging and manipulation of materials at the level of individual atoms. A successor to the STM, atomic force microscopy (AFM), works by measuring attractive or repulsive forces between the tip and the sample, and converting the basic displacement information of this tip into pictures of atoms on or in surfaces. The AFM can work with the tip touching the sample (contact mode), or the tip can tap across the surface (tapping mode). Other SPM's include the lateral-force microscope (LFM) to measure surface microfriction, magnetic force microscopes (MFM) to detect the orientation of magnetic domains, and a force-modulation microscope (FMM) to image differences in elastic moduli on the micro-scale. A very recent adaptation of the SPM probes the differences in chemical forces across a surface at the molecular scale and has been called the chemical force microscope (CFM). These developments opened the door for significant advances in material characterization 


\section{Materials Testing}

The light microscope and electron microscope are strictly imaging devices, while the probe microscopes have some utility as imaging devices and in manipulation or characterization of materials. However, to date, the accuracy and repeatability of basic force/displacement measurements taken using probe microscopy has been a subject of debate. Because of this uncertainty, it appears that accurate, quantitative material testing is currently limited to devices that resolve only down to the micro-scale $\left(10^{-6}\right.$ meters). Examples of commercial devices that operate at this resolution are nanoindentors and mini-scale test devices built by using micro-electro-mechanical-systems (MEMS). These devices can be constructed with a high degree of repeatability and will operate under a range of environmental conditions. For example, the nanoindentor is a high-precision instrument for the determination of the localized mechanical properties of thin films, coatings and substrates. An indenter tip, normal to the sample surface, with a known geometry, is driven into the sample by applying an increasing load up to some preset value. The load is then gradually decreased until partial or complete relaxation of the sample has occurred. The load and displacement are recorded continuously throughout this process from which the mechanical properties such as hardness, Young's modulus, and viscoelastic constants can be calculated. A typical nanoindentor has a depth resolution $0.02 \mathrm{~nm}$, a maximum indentation depth of $500,000 \mathrm{~nm}$, and maximum load of $500 \mathrm{mN}$ with a resolution of $50 \mathrm{nN}$.

\section{Information Technology}

The final technology element that has helped drive the advance in Computational Materials is the revolution in Information Technology (IT). In part, the IT revolution has been facilitated by the rapid increase in processing speed and power available to both desktop and mainframe computers. To illustrate this growth, one can consider Moore's Law, a prediction that forecasted processing speed to double every 18 months. The observation was made in 1965 by Gordon Moore, co-founder of Intel, and was based on the fact that the number of transistors per square inch on integrated circuits had doubled every year since the integrated circuit was invented. To date, this forecast has held true and most experts, including Moore himself, expect Moore's Law to hold for at least another two decades. These increases in processing speed have in turn helped drive the availability of software that can solve the complex problems associated with computational chemistry and continuum mechanics with increased accuracy.

\section{Structure-Property Relationships}

In order to apply modeling and computer simulation to enhance the development of nanostructured materials systems, it is necessary to consider the structure-property relationships. These relationships relate the intrinsic structure of the material to the desired engineering-level property or performance. A list of these structure-property relationships for polymers and polymer/nanotube composites is given in table 3. This table breaks down the structure according to scale and includes structure that can be directly influenced by material-synthesis methods. This table is by no means an exhaustive list but it does describe the principal structure-property elements in use by the Computational Materials program [9]'[10]. The simulation methods that address these structure-property relationships and are used to establish the multiscale modeling are molecular dynamics, coarse graining - Monte Carlo, micromechanics, and finite elements. Before outlining the simulation methods, a few key terms require definition. A Model is the simplified part of real structure. A Theory is the framework by which physical results can be predicted. A Simulation is a numerical solution. An Experiment is performed to establish the relationship between several physically connected parameters. A Measurement is the physical features observed in an experiment.

\section{Simulation Methods}

\section{Atomistic, Molecular Methods}

The approach taken by the Computational Materials Program is formulation of a set of integrated predictive models that bridge the time and length scales associated with material behavior from the nano 
through the meso scale. At the atomistic or molecular level, the reliance is on molecular mechanics, molecular dynamics, and coarse-grained, Monte-Carlo simulation. Molecular models encompassing thousands and perhaps millions of atoms can be solved by these methods and used to predict fundamental, molecular level material behavior. The methods are both static and dynamic. For example, molecular mechanics can establish the minimum-energy structure statically and molecular dynamics can resolve the nanosecond-scale evolution of a molecule or molecular assembly. These approaches can model both bonded and nonbonded forces (e.g., Van der Waals and electrostatic) but cannot explicitly account for bond cleavage.

The molecular dynamics (MD) method was first introduced by Alder and Wainwright in the late 1950 's to study the interactions of hard spheres[11],[12]. Many important insights concerning the behavior of simple liquids emerged from their studies. The next major advance was in 1964, when Rahman carried out the first simulation by using a realistic potential for liquid argon[13]. In the current literature, one routinely finds molecular dynamics simulations of organic and inorganic material systems addressing a variety of issues including the thermodynamics of biological process, polymer chemistry and crystal structure[14][15]. The number of simulation techniques has greatly expanded; there exist now many specialized techniques for particular problems, including mixed quantum mechanical - classical simulations. Molecular dynamics simulation techniques are widely used to help interpret experimental results from X-ray crystallography and nuclear magnetic resonance spectroscopy. Recent examples of atomistic simulations of carbon nanotube behavior at the nano-scale include Refs.[16] and [17].

Molecular dynamics simulations generate information at the nano-level, including atomic positions and velocities. The conversion of this information to macroscopic observables such as pressure, energy, heat capacities, etc., requires statistical mechanics. An experiment is usually made on a macroscopic sample that contains an extremely large number of atoms or molecules, representing an enormous number of conformations. In statistical mechanics, averages corresponding to experimental measurements are defined in terms of ensemble averages. For example, the average potential energy of the system is defined as

$$
V=\frac{1}{M} \sum_{i=1}^{M} V_{i}
$$

where $M$ is the number of configurations in the molecular dynamics trajectory and $V_{i}$ is the potential energy of each configuration. Similarly, the average kinetic energy is given by

$$
K=\frac{1}{M} \sum_{j=1}^{M}\left\{\sum_{i=1}^{N} \frac{m_{i}}{2} v_{i} \bullet v_{i}\right\}_{j}
$$

where $M$ is the number of configurations in the simulation, $N$ is the number of atoms in the system, $m_{i}$ is the mass of the particle $i$ and $v_{i}$ is the velocity of particle $i$. To ensure a proper average, a molecular dynamics simulation must account for a large number of representative conformations.

By using Newton's second law to calculate a trajectory, one only needs the initial positions of the atoms, an initial distribution of velocities and the acceleration, which is determined by the gradient of the potential energy function. The equations of motion are deterministic; i.e., the positions and the velocities at time zero determine the positions and velocities at all other times, $t$. In some systems, the initial positions can be obtained from experimentally determined structures.

In a molecular dynamics simulation, the time dependent behavior of the molecular system is obtained by integrating Newton's equations of motion. The result of the simulation is a time series of conformations or the path followed by each atom. Most molecular dynamics simulations are performed under conditions of constant number of atoms, volume, and energy $(\mathrm{N}, \mathrm{V}, \mathrm{E})$ or constant number of atoms, temperature, and pressure $(\mathrm{N}, \mathrm{T}, \mathrm{P})$ to better simulate experimental conditions. The basic steps in the MD simulation are given as follows.

1. Establish initial coordinates.

2. Minimize the structure. 

3. Assign initial velocities.
4. Establish heating dynamics.
5. Perform equilibration dynamics.
6. Rescale the velocities and check if the temperature is correct.
7. Perform dynamic analysis of trajectories.

Current generation force fields (or potential energy functions) provide a reasonably good compromise between accuracy and computational efficiency. They are often found empirically and calibrated to experimental results (e.g. X-ray crystallography) and quantum mechanical calculations of small model compounds. The development of parameter sets that define these force fields may require extensive optimization and is an area of continuing research. One of the most important limitations imposed on a force field is that no drastic changes in electronic structure are allowed, i.e., no events like bond making or breaking can be modeled.

The most time consuming part of a molecular dynamics simulation is the calculation of the nonbonded terms in the potential energy function, e.g., the electrostatic and van der Waals forces. In principle, the non-bonded energy terms between every pair of atoms should be evaluated. This requirement would imply that the number of computations increases as the square of the number of atoms for a pair-wise model. To speed up the computation, the interactions between two atoms separated by a distance greater than a pre-defined distance, the cutoff distance, are ignored.

\section{Monte Carlo Simulation}

Although molecular dynamics methods provide the kind of detail necessary to resolve molecular structure and localized interactions, this fidelity comes with a price. Namely, both the size and time scales of the model are limited by numerical and computational boundaries. To help overcome these limitations, coarse-grained methods are available that represent molecular chains as simpler, bead-spring models. A comparison to MD has shown up to four orders of magnitude decrease in CPU time through the use of the simpler models[18]. Although the coarse-grain models lack the atomistic detail of MD, they do preserve many of the important aspects of the chemical structure and allow for simulation of material behavior above the nano-scale[19]'[20]. The connection to the more detailed atomistic model can be made directly through an atomistic-to-coarse-grain mapping procedure that when reversed allows one to model wellequilibrated atomistic structures by performing this equilibration by using the coarse-grain model. This mapping and reverse mapping helps to overcome the time-scale upper limits of MD simulations.

Several approaches to coarse graining have been proposed and include both continuous and lattice models. The continuous models seem to be preferable for dynamic problems such as might occur when considering dynamic changes in volume[19]. As outlined in Kremer[19], the systematic development of the coarse-grain model requires three principal steps.

1. Determine the degree of coarse-graining and the geometry of the model.

2. Choose the form of the intra- and interchain potentials.

3. Optimize the free parameters, especially for the non-bonded interactions.

Coarse-grain models are often linked to Monte Carlo (MC) simulations to provide a timely solution. The MC method is used to simulate stochastic events and provide statistical approaches to numerical integration[21]. As given by Raabe[22], there are three characteristic steps in the MC simulation that are given as follows.

1. Translate the physical problem into an analogous probabilistic or statistical model.

2. Solve the probabilistic model by a numerical sampling experiment.

3. Analyze the resultant data by using statistical methods.

Monte Carlo simulation methods are roughly grouped into four categories: Weighted and nonweighted sampling methods, lattice type, spin model, and energy operator. 


\section{Continuum Methods}

Despite the importance of understanding the molecular structure and nature of materials, at some level in the multi-scale analysis the behaviour of collections of molecules and atoms can be homogenized. At this level, the continuum level, the observed macroscopic behaviour is explained by disregarding the discrete atomistic and molecular structure and assuming that the material is continuously distributed throughout its volume. The continuum material is assumed to have an average density and can be subjected to body forces such as gravity and surface forces such as the contact between two bodies.

The continuum can be assumed to obey several fundamental laws. The first, continuity, is derived from the conservation of mass. The second, equilibrium, is derived from momentum considerations and Newton's second law. The third, the moment of momentum principle, is based on the model that the time rate of change of angular momentum with respect to an arbitrary point is equal to the resultant moment. The next two laws, conservation of energy and entropy are based on the first and second laws of thermodynamics, respectively. These laws provide the basis for the continuum model and must be coupled with the appropriate constitutive equations and equations of state to provide all the equations necessary for solving a continuum problem. The state of the continuum system is described by several thermodynamic and kinematic state variables. The equations of state provide the relationships between the non-independent state variables.

The continuum method relates the deformation of a continuous medium to the external forces acting on the medium and the resulting internal stress and strain. Computational approaches range from simple closed-form analytical expressions to micromechanics to complex structural mechanics calculations based on beam and shell theory. The continuum-mechanics methods rely on describing the geometry, (i.e., a physical model), and must have a constitutive relationship to achieve a solution[23]. For a displacement based form of continuum solution, the principle of virtual work is assumed valid. In general, this is given as:

$$
\begin{aligned}
& \delta W=\oiint_{V} \sigma_{i j} \delta \varepsilon_{i j} d V \\
& =\oiiint \int_{V} P_{j} \delta u_{j} d V+\oiiint \int_{S} T_{j} \delta u_{j} d S+F_{j} \delta u_{j}
\end{aligned}
$$

where $W$ is the virtual work which is the work done by imaginary or virtual displacements, $\varepsilon$ is the strain, $\sigma$ is the stress, $P$ is the body force, $u$ is the virtual displacement, $T$ is the tractions and $F$ is the point forces. The symbol $\delta$ is the variational operator designating the virtual quantity[24]'[22]. For a continuum system, a necessary and sufficient condition for equilibrium is that the virtual work done by sum of the external forces and internal forces vanish for any virtual displacement[24].

Micromechanics

Homogenization of a multi-constituent material requires the combination of the continuum method and a micromechanics model to provide a transition from the microscale to the macroscale. Micromechanics assumes small-deformation continuum mechanics as outlined in the preceding section. Continuum mechanics, in general, assumes uniform material properties within the boundaries of the problem. At the microscale, this assumption of uniformity may not hold and hence the micromechanics method is used to express the continuum quantities associated with an infinitesimal material element in terms of the parameters that characterize the structure and properties of the micro-constituents of the element [25].

A central theme of micromechanics models is the development of a representative volume element (RVE) that is a statistical representation of the local continuum properties. In this sense, the RVE may include material boundaries, voids, and defects apparent at the microscale. The RVE is constructed to ensure that the length scale is consistent with the smallest constituent that has a first-order effect on the macroscopic behavior. The RVE is then used in a repeating or periodic nature in the full-scale model. The approach to the micromechanics solution therefore requires a RVE and a suitable averaging technique. As given in Ref.[25], the volume average of a typical, spatially variable, integrable quantity $T(x)$ is 


$$
\langle T\rangle \equiv \frac{1}{V} \int_{V} T(x) d V
$$

where $V$ is the volume of the RVE. Then, the unweighted volume average stress and strain are given by

$$
\bar{\sigma} \equiv\langle\sigma\rangle \text { and } \bar{\varepsilon} \equiv\langle\varepsilon\rangle
$$

respectively. The principle of virtual work is assumed to be valid. The micromechanics method can account for interfaces between constituents, discontinuities, and coupled mechanical and non-mechanical properties.

\section{Finite Element Methods}

Finite element methods (FEM) have a long history of development for a wide variety of applications including problems in mechanical, biological, and geological systems. The FEM goal is to provide a numerical, approximate solution to initial-value and boundary-value problems including time-dependent processes. The method uses a variational technique for solving the differential equations wherein the continuous problem described by the differential equation is cast into the equivalent variation form and the solution is found to be a linear combination of approximation functions[26][22]. In the FEM, the physical shape of the domain of interest is broken into simple subdomains (elements) that are interconnected and fill the entire domain without overlaps. A displacement-based form of the FEM starts with the principle of virtual work for a continuum described above. The following steps outline the FEM approach:

1. Replace the continuum domain with an assemblage of subdomains.

2. Select the appropriate constitutive laws.

3. Select the interpolation functions necessary to map the element topology.

4. Describe the problem by using the variational principle and divide the system level integral into subintegrals over the elements.

5. Replace continuum state variables by interpolation functions.

6. Assemble element equations.

7. Assemble global system equations.

8. Solve global system of equations, taking into account the prescribed boundary conditions.

9. Calculate the state equation values from state variables.

\section{Effective Continuum}

The current approach for connecting atomistic models to continuum models uses relevant input from the atomistic simulations and attempts to carry forward the critical information that represents the continuum with the intrinsic nano-scale features incorporated as well.An example of this connection of the atomistic description to the continuum model is the effective - continuum model of Odegard et al.[27][28]. This model recognizes that at the nanometer length scale the constituent materials such as polymers and SWNT's closely resemble an atomic lattice structure composed of discrete elements rather than a continuum. Therefore, an equivalent-continuum model of the representative volume element (RVE) is developed to facilitate bulk constitutive modeling of the composite. For the nanotube/polymer composite, a constitutive model is thus desired that will take into account the discrete nature of the atomic interactions at the nanometer length scale and the interfacial characteristics of the nanotube and surrounding polymer matrix. To formulate this constitutive model, the first step is to obtain an atomistic model of the equilibrium molecular structure of the constituents by using molecular dynamics. The molecular potential energy for the nano-structured system is described by the sum of the individual energy contributions. 


$$
E^{m}=\sum_{\text {bond }} E^{\rho}+\sum_{\text {bond }} E^{\theta}+\sum_{\text {bond }} E^{\tau}+\sum_{\text {bond }} E^{(\omega}+\sum_{\text {bond }} E^{n b}
$$

where $E^{\uparrow}, E^{0}, E^{\tau}$, and $E^{(0)}$ are the energies associated with bond stretching, angle variation, torsion, and inversion, respectively, and $E^{n b}$ is the energy of the non-bonded interactions, which includes van der Waals and electrostatic effects. For example, the specific energy terms for bond stretching, and angle variation are

$$
E^{m}=\sum_{a} K_{a}^{\rho}\left(\rho_{a}-\mathrm{P}_{a}\right)^{2}+\sum_{a} K_{a}^{\Theta}\left(\theta_{a}-\Theta_{a}\right)^{2}
$$

where the terms $\mathrm{P}_{a}$ and $\Theta_{a}$ refer to the undeformed interatomic distance of bond number $a$ and the undeformed bond-angle number $a$, respectively. The quantities $\rho_{a}$ and $\theta_{a}$ are the distance and bond-angle after stretching and angle variance, respectively. The symbols $K_{a}^{\rho}$ and $K_{a}^{\ominus}$ represent the force constants associated with the stretching and angle variance of bond and bond-angle number $a$, respectively. The individual energy contributions are summed over the total number of corresponding interactions in the molecular model.

In the second step, an equivalent-continuum model is developed in which the mechanical properties are determined based on the force constants that describe the bonded and non-bonded interactions of the atoms in the molecular model and reflect the local nanostructure. The transition from molecular model to continuum is facilitated by the selection of a representative volume element (RVE). The RVE is several nanometers in extent and thus consists of an assemblage of many atoms. As depicted schematically in Figure 2, a pin-jointed truss model that uses truss elements to represent the chemical bonds in the lattice structure may represent the RVE. The total mechanical strain energy of the truss model may take the form

$$
E^{\mathrm{t}}=\sum_{b} \sum_{a} \frac{A_{a}^{b} Y_{a}^{b}}{2 R_{a}^{b}}\left(r_{a}^{b}-R_{a}^{b}\right)^{2}
$$

where the term $r_{a}^{b}-R_{a}^{b}$ is the stretching of rod $a$ of truss member type $b$, where $R_{a}^{b}$ and $r_{a}^{b}$ are the undeformed and deformed lengths of the truss elements, respectively. This truss-model representation can then be modeled directly by using the FEM.

To develop the correspondence between the molecular and equivalent-continuum models, the total strain energies for the two models are calculated under identical loading conditions. The effective mechanical properties, or the effective geometry, of the equivalent-continuum is determined by the requirement that the strain energies be equal. The equivalent-continuum RVE can be used in a micromechanical analysis to determine the bulk constitutive properties of the composite.

\section{Modeling - Summary}

The coupled atomistic-continuum modeling approach illustrates one of the primary challenges associated with hierarchical modeling of materials; namely, the accurate prediction of physical/chemical properties and behavior from nanoscale to macroscale without loss of intrinsic structural information. The time and length scales associated with the simulation methods described in the preceding sections have been illustrated in Figure 3, with each method placed according to the upper range of its resolution. As one moves across a scale, overlaps on both time and length resolution occur, but the overall trend is consistent.

It is recognized that at each level of homogenization or scale-up, the risk of losing the key structural information increases. The way to provide an accurate check and balance against these losses is to establish verification of analysis methods and validation of simulations at both the atomic and bulk scales. 


\section{Verification and Validation}

To gain confidence in a model and to evaluate the utility of the simulation, both verification and validation need to be addressed. The American Society of Mechanical Engineers (ASME) has recently taken on this task in a Standards committee that was formed in September of 2001 on "Verification and Validation in Computational Solid Mechanics." This committee defined verification as the "process of determining that a model implementation accurately represents the developer's conceptual description of the model and the solution to the model." Essentially, this is a mathematics issue that checks whether the modeler is solving the equations correctly. Validation was defined as the "process of determining the degree to which a model is an accurate representation of the physical world from the perspective of the intended uses of the model." Therefore, validation is a physics issue that checks whether the analyst is solving the right equations.

Of course, the issues of verification and validation are not unique to Computational Materials and have been a continuous source of discussion. In a 1967 lecture on the interaction of theory and experiments, Drucker[29] stated that the purpose of experiment is to "guide the development of theory by providing the fundamental basis for an understanding of the real world." On the topic of scale, Drucker goes on to state that the continuum models could be used at the microscale but that "unless they are modified drastically, they cannot contain the information provided by experimental observation." He also warns "observations made on the free surface do not necessarily indicate what is happening throughout the bulk of the material." In a more recent paper, Knauss[30] provides the definitions and relationships among measurements, experiments, models and theory. He states "the method consists in observing physical fact(s) and formulating an analytical framework for them to produce a scheme or theory by which other physical results can be predicted" and warns against "theories or models that are ultimately no more than a demonstration of computational feasibility, without adding any really new understanding of the underlying science." On the topic of scale, Knauss notes that at the nanoscale "there will be a continuing need to simulate such large molecular structures through assumptions that need physical examination, i.e., experimentation at the nanoscale."

Schematically, these ideas, the processes of verification and validation and the relationship to measurements and experiments are illustrated in Figure 4. Although the process of verification and validation is somewhat circular, the entry point into this process is clearly through experiments that help determine the validity of theory and assumptions while also helping to quantify the state variables associated with the problem.

It is therefore necessary that the Computational Materials approach must use experimental data to establish the range of performance of a material and to validate predicted behavior. Even at the atomistic level, methods such as molecular dynamics require careful parameterization (fit) to empirical data.

Therein, perhaps, lies the biggest challenge to Computational Materials: validation of methods across the complete range of length and time scales. To achieve this validation requires advances in measurement sciences as well as advances in theory and models, coupled with integrated, interdisciplinary research. It is imperative that research laboratories maintain a focused effort to develop new programs that provide for the simultaneous growth of all the critical elements that are required for validation of multi-scale methods.

\section{Spatial Resolution of Measurement Devices}

The spatial resolution of the previously described measurement devices is an important consideration for Computational Materials. A numerical comparison of typical spatial resolution is provided in table 2 .

However, to correctly address the requirements of characterizing nanostructured materials, the timescale limits must also be taken into consideration. To put these limits in perspective, Figure 5 illustrates how the primary measurement devices used in the LaRC Computational Materials program compare on a time versus length-scale plot. In this Figure, each device is placed according to the upper range of it's resolution. As one moves across a scale, overlaps on both time and length resolution occurs, but the overall trend is consistent. An important break point on this plot occurs at the wavelength of light. At 
lengths greater than this break point, most displacement measurements are field quantities while below this break point displacement measurements are point quantities.

Overlaying Figs. 3 and 5 onto a single plot provides a comparison between the spatial and time scales of measurement and simulation. This comparison plot is shown in Figure 6. Although this comparison is somewhat subjective, the obvious result is that direct validation of molecular-scale simulation methods, such as MD, are difficult because of the limited time-scale range of the measurement methods such as electron and probe microscopy.

\section{Concluding Remarks}

Computational Materials research at the NASA Langley Research Center proposes to significantly reduce development costs of new nanostructured materials for aerospace applications by bringing physical and microstructural information into the realm of the design engineer. The intent of the program is to assist the material developer by providing a rational approach to material development and concurrently assist the structural designer by providing an integrated analysis tool that incorporates fundamental material behavior. The approach is to draw upon advances in measurement sciences, biological sciences, and information technology to develop multi-scale simulation methods that are validated by critical experiments across a wide range of time and length scales. Currently, key structureproperty relationships are being addressed by atomistic and continuum methods that include molecular dynamics, Monte-Carlo simulation, micromechanics and finite element methods. Advances to date include constitutive relationships and effective-continuum representations of polymers and polymer/nanotube composite materials. Critical issues that remain unresolved include seamless transfer of data between the nano-to-meso-scale models and experimentally validating simulations of atomistic behavior.

\section{References}

1. Feynman, R., There's Plenty of Room at the Bottom in American Physical Society, February 1960, December, 29, 1959, E.a.S. California Institute of Technology, Pasadena.

2. National Nanotechnology Initiative: The initiative and it's implementation plan. National Science and Technology Council, Committee on Technology, Subcommittee on Nanoscale Science, Engineering and Technology, Washington, D.C., 2000, July.

3. Edelstein, A.S. and R.C. Cammarata, eds. Nanomaterials: Synthesis, Properties and Applications. 1996, Institute of Physics: Bristol.

4. Kroto, H.W., et al., C60 Buckminsterfullerene, Nature, 1985, 318: p. 162.

5. Iijima, S., Helical microtubes of of graphitic carbon, Nature, 1991, 354: p. 56.

6. Harris, P.J.F., Carbon Nanotubes and Related Structures. 1999, Cambridge: Cambridge University Press.

7. Watson, J.D. and F.H.C. Crick, Molecular structure of nucleic acids, Nature, 1953, 171(4356): p. $737-8$.

8. Human Genome Program, To Know Ourselves, U.S. Department of Energy, 1996.

9. Hinkley, J.A. and J.F. Dezern, Crystallization of Stretched Polyimides: A Structure-Property Study, NASA Langley Research Center, NASA/TM-2002-211418, 2002, April.

10. Nicholson, L.M., K.S. Whitley, T.S. Gates, and J.A. Hinkley, Influence of molecular weight on the mechanical performance of a thermoplastic glassy polyimide, Journal of Material Science, 2000, 35(24): p. 6111-6121.

11. Alder, B.J. and T.E. Wainwright, Phase Transition for a Hard Sphere System, Journal of Chemical Physics, 1957, 27: p. 1208-1211.

12. Alder, B.J. and T.E. Wainwright, Studies in Molecular Dynamics. I. General Method, Journal of Chemical Physics, 1959, 31: p. 459-466.

13. Rahman, A., Correlations in the Motion of Atoms in Liquid Argon, Physical Review, 1964, 136(2A): p. 405-411.

14. Young, J.A., B.L. Farmer, and J.A. Hinkley, Molecular modeling of the poling of piezoelectric polyimides, Polymer, 1999, 40(10): p. 2787.

15. Young, J.A., J.A. Hinkley, and B.L. Farmer, Molecular Simulations of the Imidization of Adsorbed Polyamic Acid, Macromolecules, 2000, 33: p. 4936. 
16. Frankland. S.J.V., A. Caglar, D.W. Brenner, and M. Griebel. Molecular Simulation of the Influence of Chemical Cross-Links on the Shear Strength of Carbon Nanotube-Polymer Interfaces, Journal of Physical Chemistry B. 2002, 106: p. 3046-3049.

17. Saether, E., R.B. Pipes. and S.J.V. Frankland, Nanostructured composites: Effective mechanical property determination of nanotube bundles, NASA Langley Research Center, NASA/CR-2002-211461, 2002. April.

18. Lopez, C.F., et al., Computer simulation studies of biomembranes using a coarse grain model, Computer Physics Communications, 2002, 147: p. 1-6.

19. Kremer, K. and F. Muller-Plathe, Multiscale Problems in Polymer Science: Simulation Approaches, Materials Reseach Society Bulletin, 2001(March): p. 205-214.

20. Hinkley, J.A. and J.A. Young, Monte Carlo Simulation of Endlinking Oligomers, NASA Langley Research Center, NASA/TM-1998-207649, 1998, April.

21. Monte Carlo and Molecular Dynamics Simulations in Polymer Science, ed. K. Binder. 1995, New York: Oxford University Press.

22. Raabe, D., Computational Materials Science. 1998, Weinheim: Wiley-VCH.

23. Mase, G.E., Theory and Problems of Continuum Mechanics. 1970, New York: McGraw-Hill.

24. Yang, T.Y., Finite Element Structural Analysis. 1986, Englewood Cliffs: Prentice-Hall.

25. Nemat-Nasser, S. and M. Hori. Second ed, Micromechanics: Overall Properties of Heterogeneous Materials. 1999, Amsterdam: Elsevier.

26. Reddy, J.N., An Introduction to the Finite Element Method. 1984, New York: McGraw-Hill.

27. Odegard, G.M., T.S. Gates, L.M. Nicholson, and K.E. Wise, Equivalent-Continuum Modeling of NanoStructured Materials, Composites Science and Technology, 2002, 62(14): p. 1869-1880.

28. Odegard, G.M., T.S. Gates, and K.E. Wise, Consitutive Modeling of Nanotube-Reinforced Polymer Composites in 43rd AIAA/ASME/ASCE/AHS/ASC Structures. Structural Dynamics, and Materials Conference, AIAA-2002-1427, Aprill 22-25, 2002, Denver, CO.

29. Drucker, D.C., Thoughts on the Present and Future Interrelation Theoretical and Experimental Mechanics, Experimental Mechanics, 1968.

30. Knauss, W.G., Perspectives in experimental solid mechanics, International Journal of Solids and Structures, 2000, 37: p. 251-266. 
Table 1. Significant events in materials development over the last 350 years.

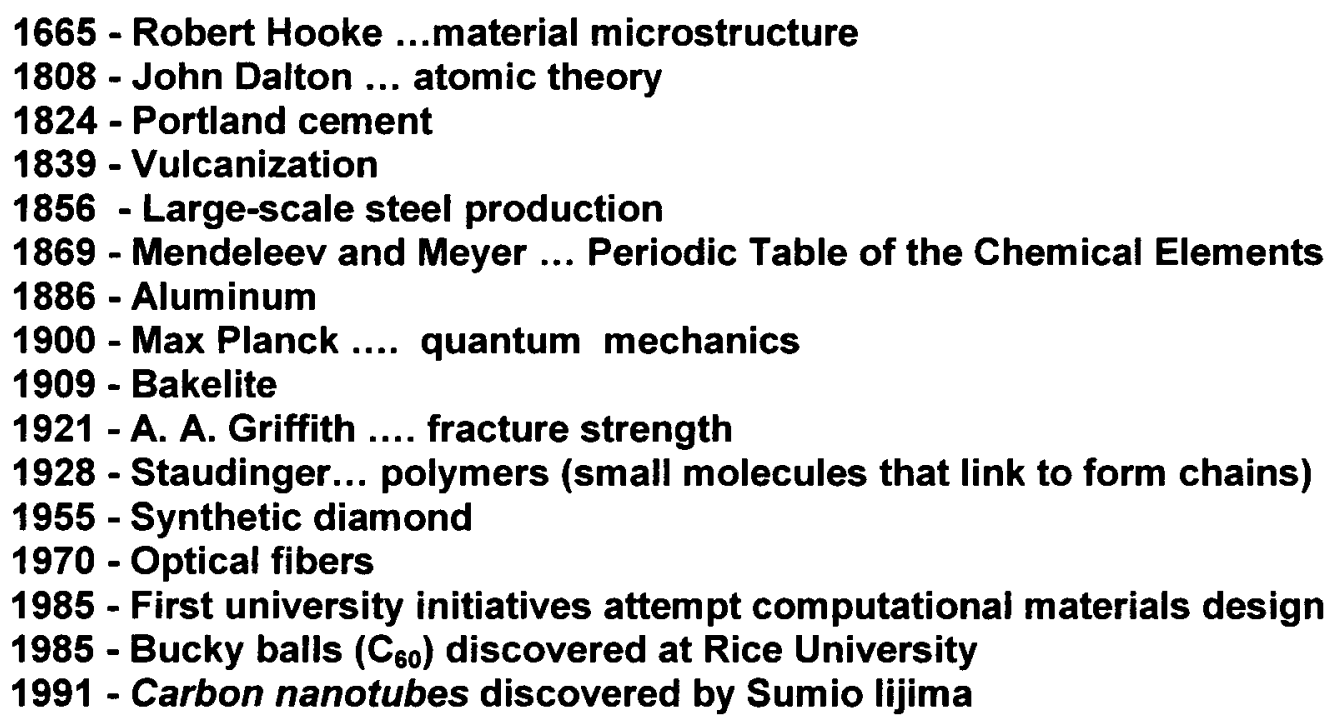

Table 2. Typical spatial resolution of devices used for material characterization and testing.

\begin{tabular}{|c|c|}
\hline Device & Spatial Resolution (nm) \\
\hline AFM & .001 \\
\hline TEM & .2 \\
\hline SEM & 5 \\
\hline Light microscope & 200 \\
\hline MEMS/nanoindentor & 250 \\
\hline
\end{tabular}


Table 3. Structure-property relationships for polymer and polymer/carbon nanotube materials.

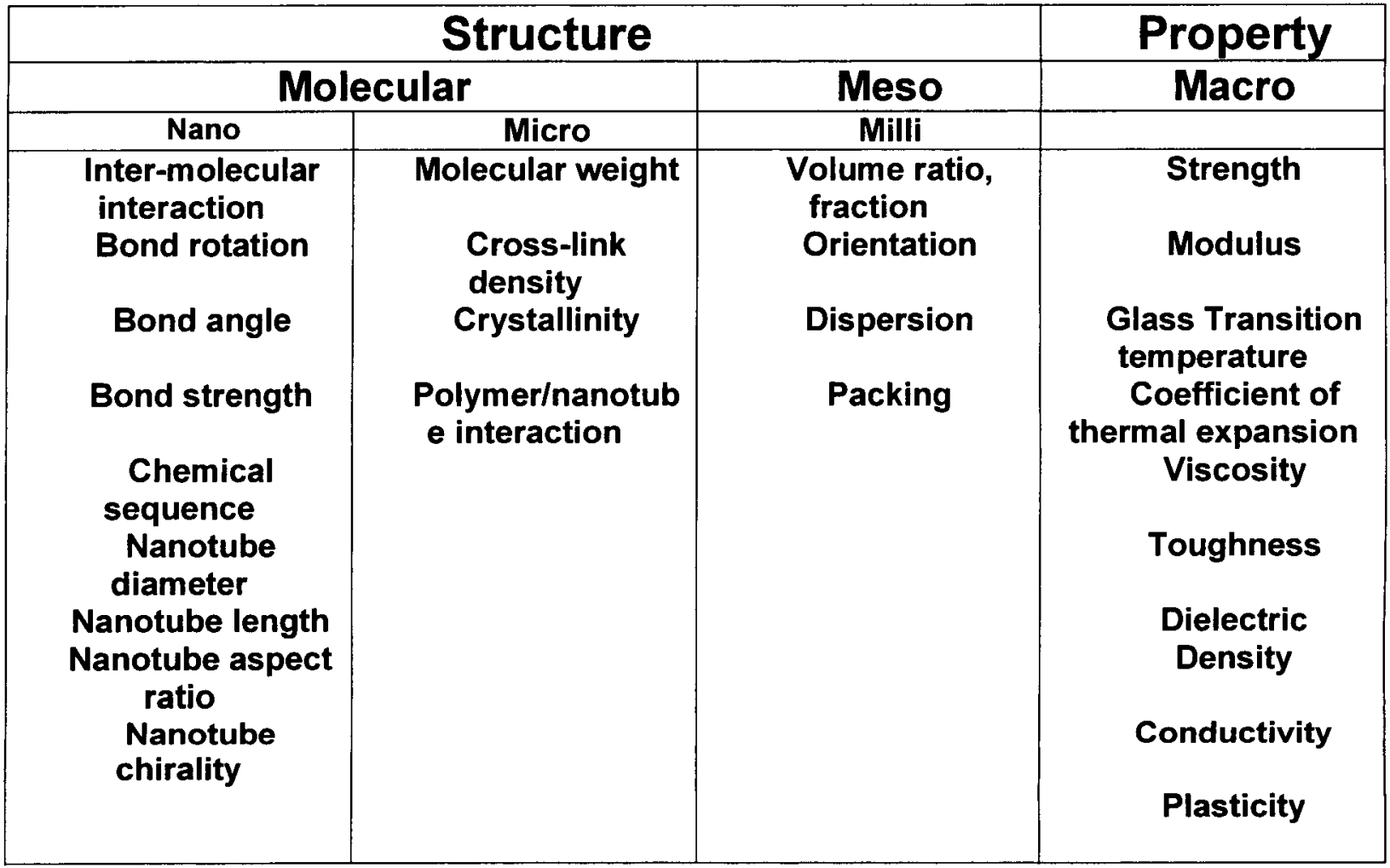

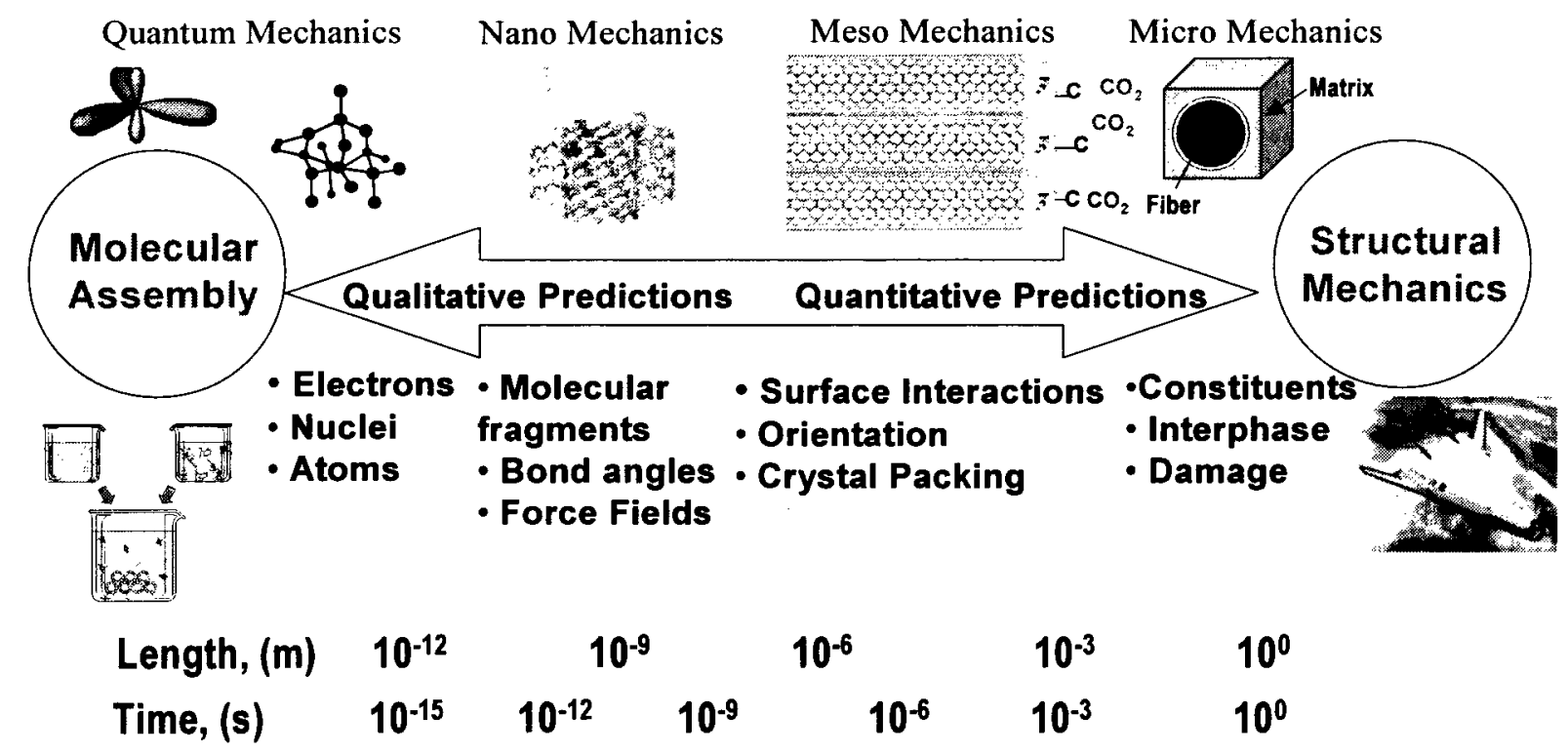

Figure 1. Schematic illustration of relationships between time and length scales for the multi-scale simulation methodology. 


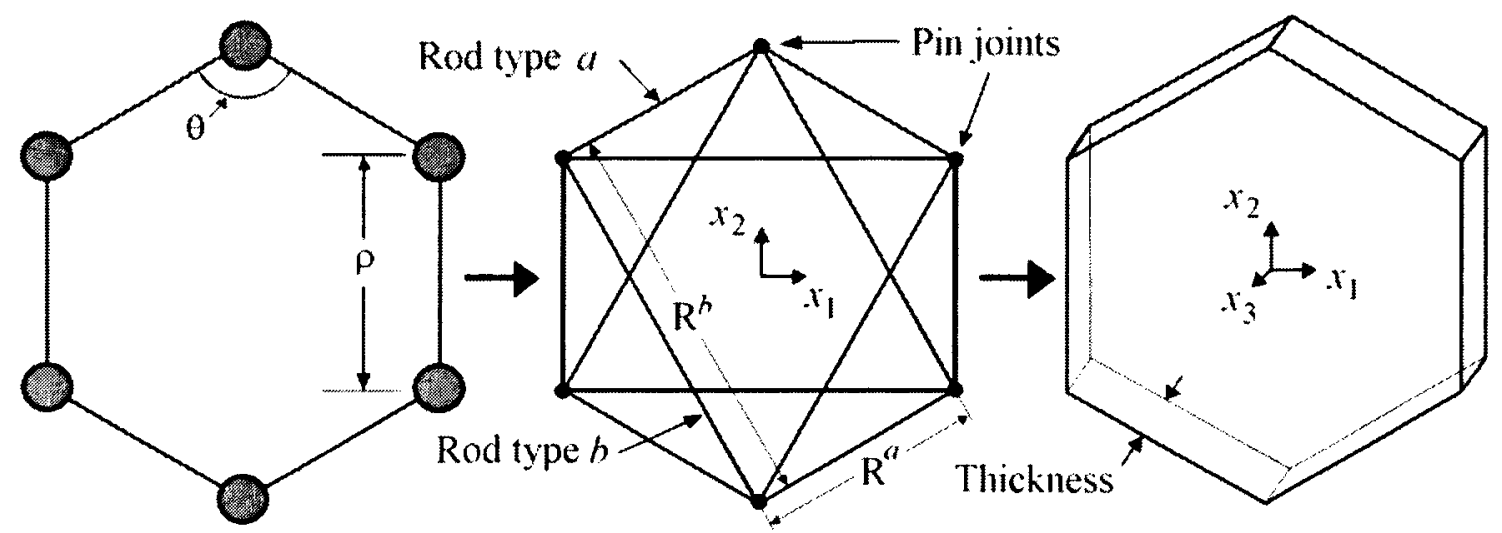

Chemical bonds

Pin-jointed truss

Continum

Figure 2. Representative volume elements for the chemical, truss, and continuum models where $\theta, \rho$, and $R$ are dimensions.

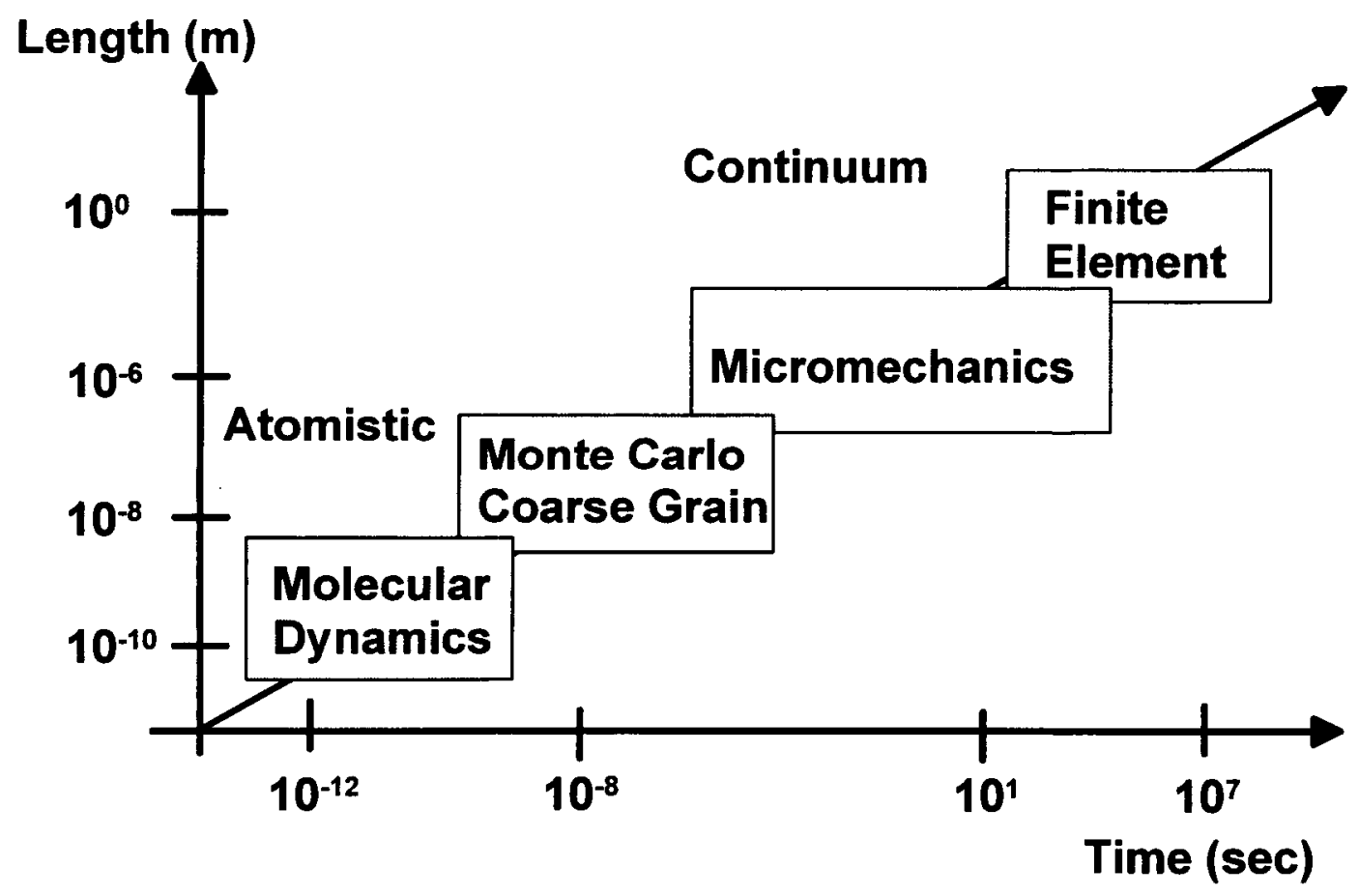

Figure 3. Range of length and time scales associated with key simulation methods. 
Determine

validity of

theory and

assumptions

Experiments

Quantify state

variables

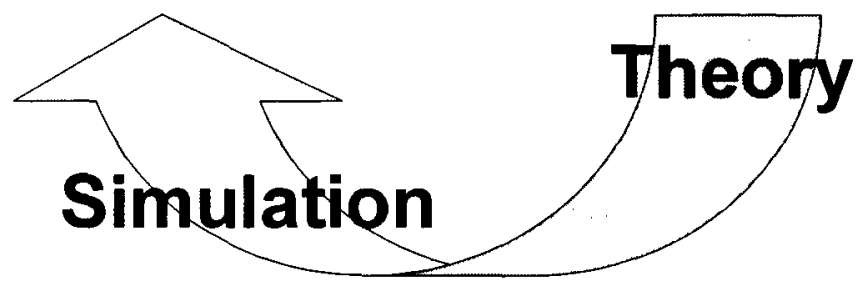

Figure 4. Illustration of the interdependence of methods and the nature of method validation and verification.

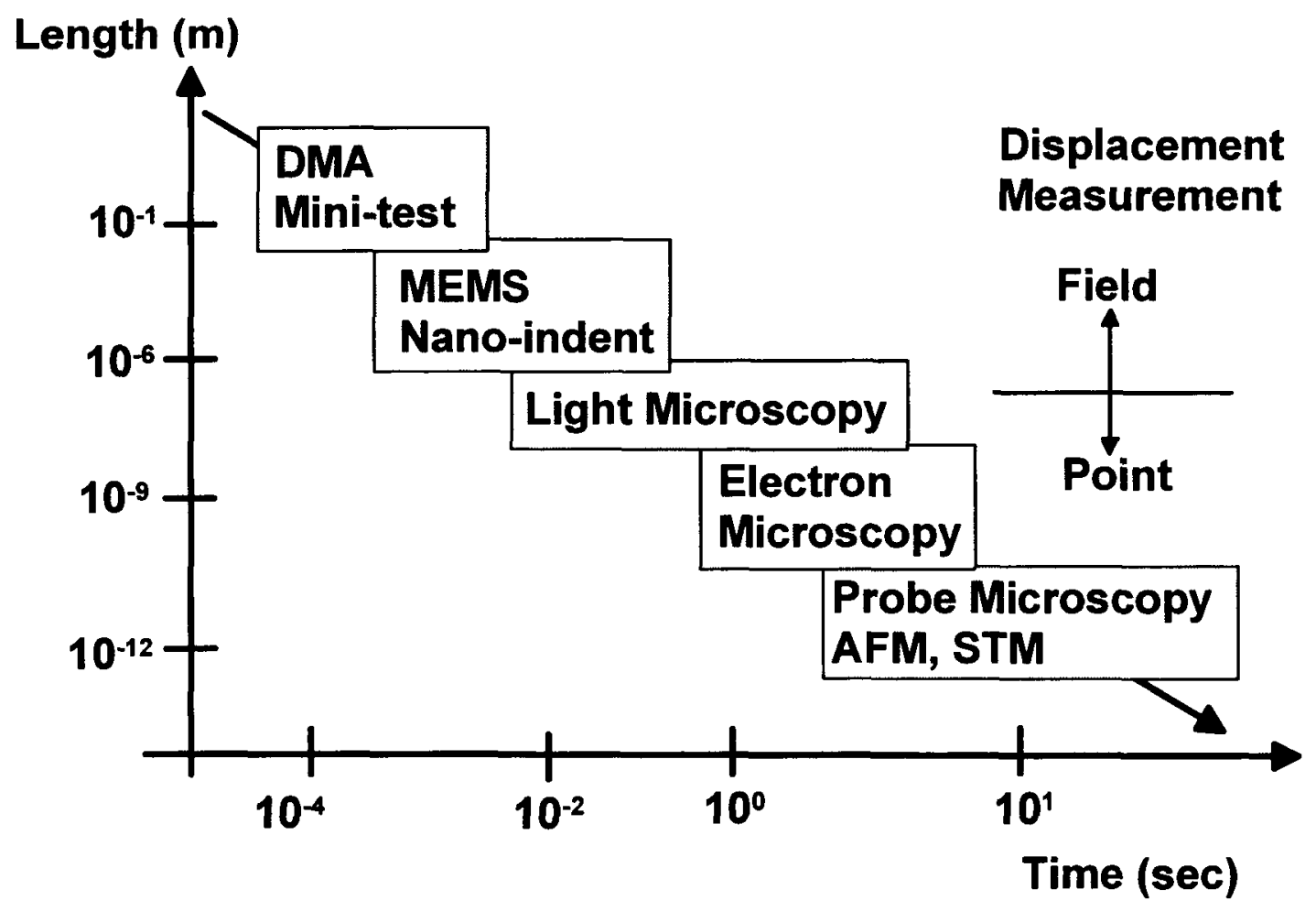

Figure 5. Range of length and time scales associated with key measurement methods. 
Length (m)

Simulation

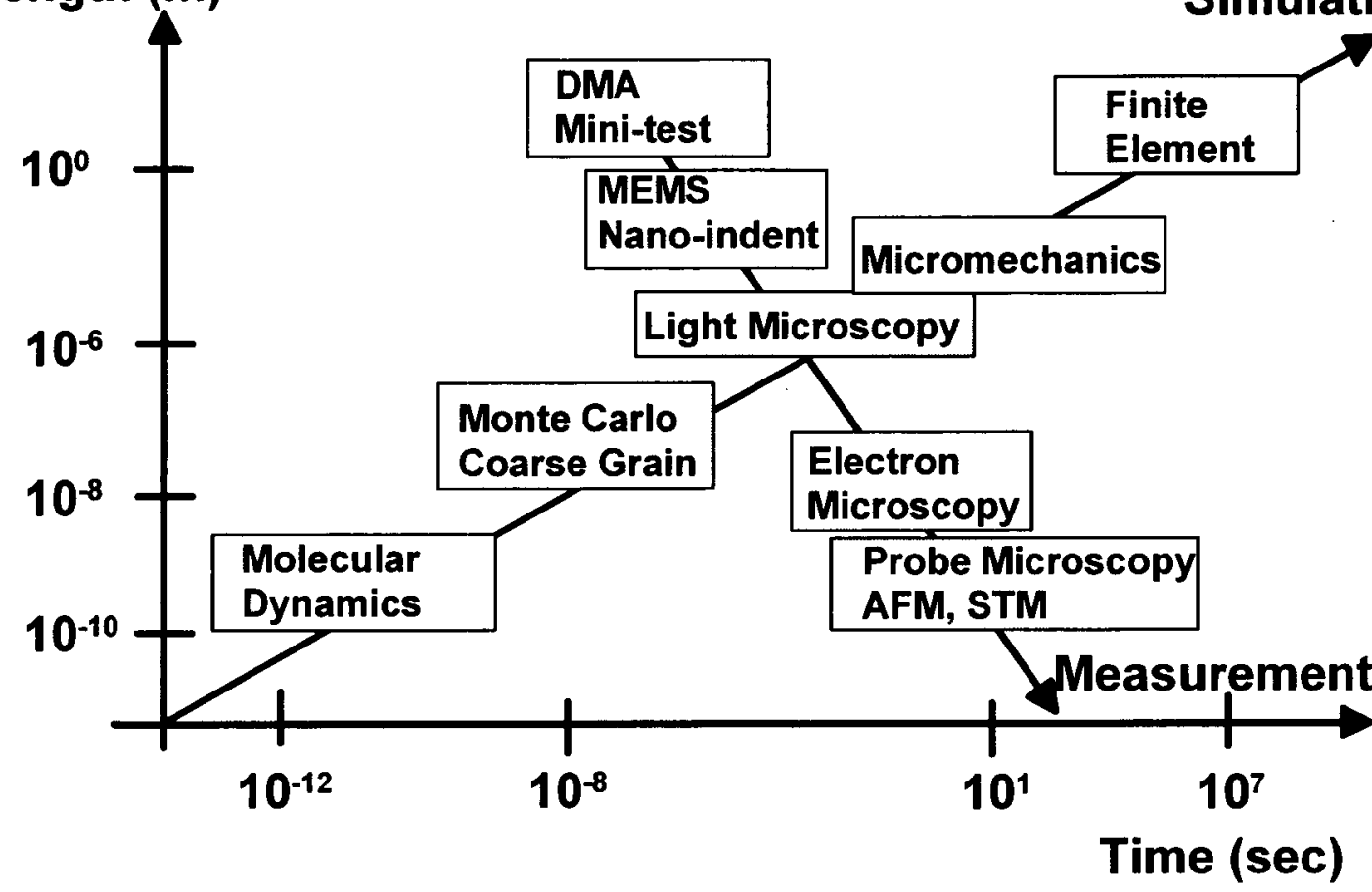

Figure 6. Illustration of the intersection of the key measurement and simulation methods from Figs. 3 and 5 respectively. 
Public reporting burden for this collection of intormation is estimated to average 1 hour per response, including the time for reviewing instructions. searching existing data sources, gathering and maintaining the data needed, and completing and reviewing the collection of information. Send comments regarding this burden estimate or any other aspect of this collection of information. including suggestions for reducing this burden, to Washington Headquarters Services. Directorate for Information Operations and Reports. 1215 Jefferson Davis Highway, Suite 1204, Arlington, VA 22202-4302, and to the Olfice of Management and Budget, Paperwork Reduction Project (0704-0188), Washington, DC 20503.

\begin{tabular}{|l|l|l}
\hline 1. AGENCY USE ONLY (Leave blank) & $\begin{array}{l}\text { 2. REPORT DATE } \\
\text { March 2003 }\end{array}$ & $\begin{array}{l}\text { 3. REPORT TYPE AND DATES COVERED } \\
\text { Technical Memorandum }\end{array}$ \\
\hline
\end{tabular}

\begin{tabular}{l|l|l|l|}
\hline 4. TITLE AND SUBTITLE & 5. FUNDING NUMBERS
\end{tabular}

Computational Materials: Modeling and Simulation of Nanostructured

Materials and Systems

$706-63-51-03$

\section{AUTHOR(S)}

Thomas S. Gates and Jeffrey A. Hinkley

\section{PERFORMING ORGANIZATION NAME(S) AND ADDRESS(ES)}

NASA Langley Research Center

Hampton, VA 23681-2199

8. PERFORMING ORGANIZATION REPORT NUMBER

L-18267

9. SPONSORING/MONITORING AGENCY NAME(S) AND ADDRESS(ES)

10. SPONSORING/MONITORING AGENCY REPORT NUMBER

National Aeronautics and Space Administration

Washington, DC 20546-0001

NASA/TM-2003-212163

\section{SUPPLEMENTARY NOTES}

\section{2a. DISTRIBUTION/AVAILABILITY STATEMENT \\ Unclassified-Unlimited \\ Subject Category $24 \quad$ Distribution: Standard \\ Availability: NASA CASI (301) 621-0390}

13. ABSTRACT (Maximum 200 words)

The paper provides details on the structure and implementation of the Computational Materials program at the NASA Langley Research Center. Examples are given that illustrate the suggested approaches to predicting the behavior and influencing the design of nanostructured materials such as high-performance polymers, composites, and nanotube-reinforced polymers. Primary simulation and measurement methods applicable to multi-scale modeling are outlined. Key challenges including verification and validation of models are highlighted and discussed within the context of NASA's broad mission objectives.

\section{SUBJECT TERMS}

multi-scale modeling, nanostructured materials, nanotechnology, simulation

12b. DISTRIBUTION CODE

\begin{tabular}{|c|c|c|}
\hline $\begin{array}{l}\text { 17. SECURITY CLASSIFICATION } \\
\text { OF REPORT } \\
\text { Unclassified }\end{array}$ & $\begin{array}{l}\text { 18. SECURITY CLASSIFICATION } \\
\text { OF THIS PAGE } \\
\text { Unclassified }\end{array}$ & $\begin{array}{l}\text { 19. SECURITY CLASSIFICATION } \\
\text { OF ABSTRACT } \\
\text { Unclassified }\end{array}$ \\
\hline
\end{tabular}

15. NUMBER OF PAGES

22

16. PRICE CODE

\section{LIMITATION}

OF ABSTRACT

UL 